\title{
GROWTH SEQUENCES OF FINITE GROUPS III
}

\author{
JAMES WIEGOLD \\ (Received 22 September; revised 18 November 1977) \\ Communicated by M. F. Newman
}

\begin{abstract}
Let $G$ be a finite group with $d(G)=\alpha, d\left(G / G^{\prime}\right)=\beta \geqslant 1$. If $G$ has non-abelian simple images, let $s$ denote the order of a smallest such image. Then $d\left(G^{n}\right)=\beta n$ provided that $\beta n \geqslant \alpha+1+\log _{8} n$. If all simple images of $G$ are abelian, then $d\left(G^{n}\right)=\beta n$ provided that $\beta n \geqslant \alpha$. If $G$ is non-trivial and perfect, with $s$ again denoting the order of a smallest non-abelian simple image, then $d\left(G^{s^{n}}\right) \leqslant d(G)+n$ for all $n \geqslant 0$. These results improve on results in previous papers with similar titles.
\end{abstract}

Subject classification (Amer. Math. Soc. (MOS) 1970): 20 D 99.

In this short note I shall give simple proofs of improvements on the results obtained in the second paper in this series (Wiegold (1975); I shall refer to this as W2, and to Wiegold (1974) as W1). To avoid repetition and to save space, I shall assume that the reader is familiar with those two articles.

I begin with the observation that Corollary 3.2 of W2 is an absurdly weak consequence of Lemma 3.1, and in fact the following stronger consequence practically stares one in the face.

LEMMA 1. For every finite perfect group B and every integer $n \geqslant 0, d\left(B^{2^{n}}\right) \leqslant d(B)+n$.

This result was noted in Gruenberg (1976), Lemma 6.18. As a matter of fact, it is very easy to give a proof exhibiting generators for $B^{2^{n}}$ in terms of generators for $B$ and an element whose normal closure is $B$, but $I$ shall not stop to do this. Lemma 1 is quite sufficient to give much better bounds than those to be found in Theorem $\mathrm{B}$ of $\mathrm{W} 2$.

However, Lemma 1 can be improved in such a way as to yield bounds that are very close to best possible:

LEMMA 2. Let $B$ be a non-trivial finite perfect group and $s$ the order of a smallest non-abelian simple image of $B$. Then $d\left(B^{s^{n}}\right) \leqslant d(B)+n$ for all integers $n \geqslant 0$; and hence $d\left(B^{n}\right)<d(B)+1+\log _{s} n$ for all $n \geqslant 1$. 
Proof. By Lemmas 1.1 and 1.2 of W2 (the first of which is based on Satz 3 of Gaschütz (1955)), it is enough to establish Lemma 2 in the case where $B$ is a nontrivial power $S^{\lambda}$ of a non-abelian simple group $S$, of order $s$; and to do that, it suffices to prove that $d\left(S^{\lambda s}\right) \leqslant d\left(S^{\lambda}\right)+1$.

Let $m$ be a positive integer, and set $h(m)=\max \left\{k: d\left(S^{k}\right) \leqslant m\right\}$. The arguments of W1 and W2 were based in part on Philip Hall's discovery of a precise formula for $h(m)$ in terms of the Möbius function of the subgroup lattice of $S$ (Hall, 1936). It now appears that the key point is the following:

For every $m \geqslant d(S), h(m+1) \geqslant \operatorname{sh}(m)$.

This is straightforward. By Hall (1936) (see Section 4 of W1), all we have to do is to prove that $\varphi(m+1) \geqslant s \varphi(m)$, where, for each $l, \varphi(l)$ denotes the number of $l$-bases of $S$, that is, ordered $l$-tuples of elements of $S$ that generate $S$. But that is transparent; given any $m$-basis, one constructs $s$ different $(m+1)$-bases by tacking on elements of $S$ at the end. Different $m$-bases give rise to different $(m+1)$-bases in this way.

To prove that $d\left(S^{\lambda_{s}}\right) \leqslant d\left(S^{\lambda}\right)+1$, set $d\left(S^{\lambda}\right)=m$. Then $m \geqslant d(S)$ and $\lambda \leqslant h(m)$. Thus

$$
d\left(S^{\lambda s}\right) \leqslant d\left(S^{h(m) s}\right) \leqslant d\left(S^{h(m+1)}\right)=m+1,
$$

which gives us what we want.

Note that Lemma 2 is a strengthened version of Theorem A of W2, and that it has been obtained very cheaply. The main result of the paper, Theorem 3 following, is a similarly strengthened version of Theorem B. I am very grateful to the referee of this paper, who pointed out that I had overlooked part (b).

THEOREM 3. Let $G$ be a finite imperfect group with $d(G)=\alpha, d\left(G / G^{\prime}\right)=\beta$.

(a) Let $s$ be the order of a smallest non-abelian simple image of $G$, if any such images exist. Then $d\left(G^{n}\right)=n$ provided that $n \geqslant(1 / \beta)\left(\alpha+1+\log _{s} n\right)$.

(b) If all simple images of $G$ are abelian, then $d\left(G^{n}\right)=\beta n$ provided that $n \geqslant \alpha / \beta$.

The proof is a close copy of that on p. 228 of W2, with the extra ingredient of Lemma 2. Since $d\left(G^{n}\right) \geqslant \beta n$ in all cases, our task is to consider the reverse inequality. As in $\mathrm{W} 2$, let $R(G)$ be the intersection of the maximal normal subgroups of $G$, and set $G_{*}=G / R(G)$. By Lemma 1.1 of W $2, d\left(G^{n}\right) \leqslant \beta n$ if $d(G) \leqslant \beta n$ and $d\left(G_{*}^{n}\right) \leqslant \beta n$. But $G_{*}=A \times B$, where $A$ is an abelian group with $d(A)=\beta$, and $B$ is perfect. Since $G_{*}^{n}=A^{n} \times B^{n}$ and $d\left(A^{n}\right)=\beta n$, part (b) now follows immediately, because $B$ is trivial in that case. For part (a), observe that Lemma 5.1 of $\mathrm{W} 1$ gives that $d\left(G_{*}^{n}\right)=\max \left(d\left(A^{n}\right), d\left(B^{n}\right)\right)$, so that $d\left(G^{n}\right) \leqslant \beta n$ provided that $d\left(B^{n}\right) \leqslant \beta n$ and $d(G) \leqslant \beta n$. By Lemma $2, d\left(B^{n}\right) \leqslant d(B)+1+\log _{s} n$ for all $n \geqslant 1$, while $d(B) \leqslant \alpha$ since $B$ is an image of $G$. Putting all these facts together, we get that $d\left(G^{n}\right) \leqslant \beta n$ whenever $\beta n \geqslant \alpha+1+\log _{s} n$, as required. 
I believe that Theorem 3 is about as close as one will get to best possible bounds using such simple-minded ideas as I have. Indeed, as we shall see soon, it is really not far out. One way in which it differs from Theorem B is that part (a) does not prescribe a bound $f(\alpha, \beta)$ such that $d\left(G^{n}\right)=\beta n$ for $n \geqslant f(\alpha, \beta)$. However, it is very simple indeed to do this. To aid the exposition, let us agree that from now on we consider only groups that do have non-abelian simple images, and that $s$ will denote the order of a smallest one for the relevant group. This is, after all, the more interesting case.

COROLlaRy 4. $d\left(G^{n}\right)=\beta n$ provided that $n \geqslant(1 / \beta)\left(\alpha+1+2 \log _{s} \alpha\right)$.

A further simple arithmetical calculation yields the following result, which I state in a form that brings out the contrast with the counterexamples given in Theorem 4.1 of W2:

Corollary 5. For every real number $b>1$, there exists an integer $\alpha^{\prime}$ such that, if $G$ is an imperfect group with $d(G)=\alpha, d\left(G / G^{\prime}\right)=\beta$ and $\alpha \geqslant \alpha^{\prime}$, then $d\left(G^{n}\right)=\beta n$ provided that $n \geqslant(1 / \beta)\left(\alpha+b \log _{s} \alpha\right)$.

Of course, some of the information in Theorem 3 is lost in these corollaries.

Perhaps it would be as well to spell out the essential content of Theorem 4.1 just mentioned, so that the reader may see the contrast. For every positive real number $c$ with $c<1$ and every integer $\beta \geqslant 1$, there exists an integer $\alpha^{\prime}$ such that, for all $\alpha \geqslant \alpha^{\prime}$, there exists a finite group $G$ with $d(G)=\alpha, d\left(G / G^{\prime}\right)=\beta$ and $d\left(G^{n}\right)>\beta n$ for $n \leqslant(1 / \beta)\left(\alpha+c \log _{s} \alpha\right)$.

\section{REFERENCES}

W. Gaschütz (1955), "Zu einem von B. H. und H. Neumann gestellten Problem", Math. Nachr. 14, 249-252.

K. W. Gruenberg (1976), Relation Modules of Finite Groups, Regional Conference Series in Mathematics, 25 (American Mathematical Society, Providence, R.I.).

P. Hall (1936), "The Eulerian functions of a group", Quart. J. Math. Oxford 7, 134-151.

J. Wiegold (1974), "Growth sequences of finite groups", J. Austral. Math. Soc. 17, 133-141.

J. Wiegold (1975), "Growth sequences of finite groups II", J. Austral. Math. Soc. 20, 225-229.

University College

Cardiff, Wales 